\title{
Diseño y construcción de mobiliario escolar portátil y sustentable: experiencia de trabajo interdisciplinar en semilleros de investigación
}

Design and construction of portable and sustainable school furniture: Interdisciplinary work experience in research hotbed

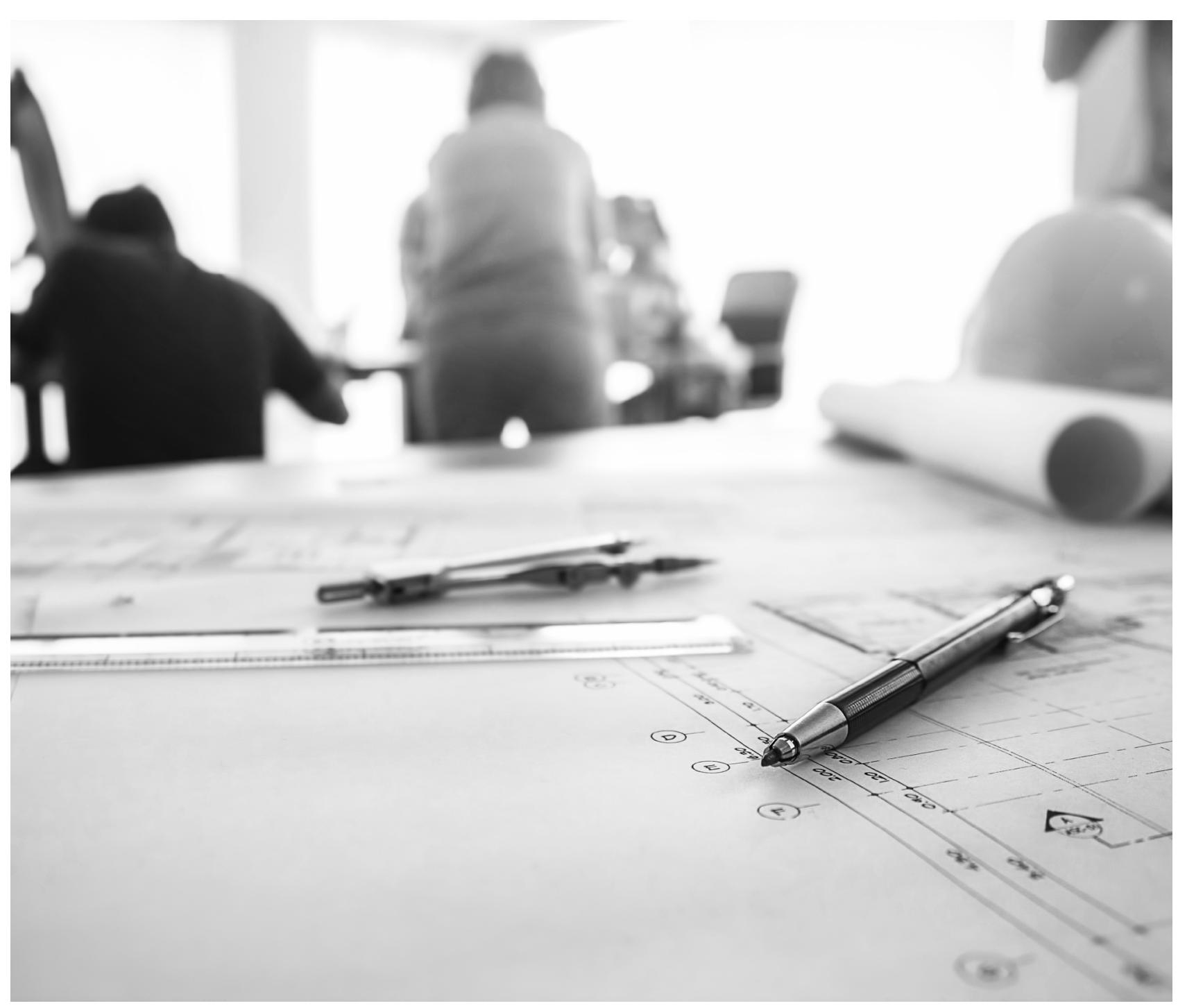




\title{
Diseño y construcción de mobiliario escolar portátil y sustentable: experiencia de trabajo interdisciplinar en semilleros de investigación ${ }^{1}$ Design and construction of portable and sustainable school furniture: Interdisciplinary work experience in research hotbed
}

\author{
Gonzalo Ramírez Gómez², Viviana Guevara ${ }^{3}$, Hernán Villamizar ${ }^{4}$
}

Artículo recibido en abril de 2018; artículo aceptado en agosto de 2018.

Este artículo puede compartirse bajo la Licencia Creative Commons Atribución-NoComercial-Compartirlgual 4.0 Internacional

y se referencia usando el siguiente formato: Ramírez Gómez, G., Guevara, V. \& Villamizar, H. (2019). Diseño y construcción

de mobiliario escolar portátil y sustentable: experiencia de trabajo interdisciplinar en semilleros de investigación.

I+D Revista de Investigaciones, 13 (1), 7-13. DOI: https://doi.org/10.33304/revinv.v13n1-2019001

\begin{abstract}
Resumen
Este artículo presenta los resultados del trabajo interdisciplinar de los semilleros de investigación DICUBO, que estudia diversos problemas en torno al diseño industrial, y el Semillero Innume, que se encarga de desarrollos relativos al diseño gráfico. El proyecto se propuso el diseño de un mobiliario escolar portátil y sustentable dirigido a niños en primer grado de escolaridad de instituciones educativas con escasos recursos del Área Metropolitana de Bucaramanga. Además, el producto se planteó como parte de un proceso de apropiación del objeto por parte del usuario; se determinó que el mueble funcionara como objeto lúdico y mediador de aprendizajes significativos en cuanto a ordenación espacial, ecología, funcionalidad e intervención gráfica artística. En el desarrollo de este proyecto se entrecruzaron diversas disciplinas con el aporte de sus métodos propios como la filosofía del Lean Startup y la etnografía; el proceso de diseño se llevó a cabo de acuerdo a la metodología de design thinking, tanto en el aspecto gráfico como en el diseño industrial. Este trabajo se fundamentó en el diseño incluyente para la formación integral de niños que requieren atención en su educación; el producto permite no solo contar con un mobiliario, sino desarrollar procesos cognitivos básicos para la edad de seis años, el desarrollo de habilidades y procesos lógicos de reconocimiento.
\end{abstract}

Palabras clave: diseño gráfico, diseño industrial, escolar, mobiliario, sustentable.

\section{Abstract}

This article presents the results of the interdisciplinary work of the DICUBO research hotbed, which studies problems

1. Artículo de investigación científica de enfoque cualitativo, resultado de un proyecto de investigación culminado, perteneciente al área de Diseño, Comunicación y Artes, desarrollado en el grupo de investigación Paloseco, semilleros de Investigación Dicubo e Innume. Fue financiado por la Universidad de Investigación y Desarrollo (UDI), Bucaramanga (Colombia). Dirección Calle 9 No. 23-55, PBX: 6352525. Fecha de inicio: enero de 2014. Fecha de terminación: diciembre de 2015.

2. Vinculado al Grupo de investigación INTERFAZ, Escuela de Diseño Industrial, Universidad Industrial de Santander (Bucaramanga, Colombia). Dirección: Carrera 27 calle 9, PBX: 6344000. ORCID ID: https://orcid.org/0000-0002-7343-418X. Correo electrónico: gonzaramirezg@gmail.com.

3. Diseñadora Gráfica, Especialista en Diseño Publicitario, Universidad de Investigación y Desarrollo. Vinculada a la Universidad de Investigación y Desarrollo (Bucaramanga, Colombia). Dirección: Calle 9 No. 23-55, PBX: 6352525. ORCID ID: https://orcid.org/0000-0001-8072-5666. Correo electrónico: guevaranavarro@gmail.com.

4. Grupo de Investigación Paloseco, Universidad de Investigación y Desarrollo (Bucaramanga, Colombia). Dirección: Calle 9 No. 23-55, PBX: 6352525. ORCID ID: https://orcid.org/0000-0001-5363-8057. Correo electrónico institucional: hvillamizar1@udi.edu.co 
related to industrial design and the INNUME Seedbed, which is responsible for carrying out the graphic design analysis. The project proposed the design of a portable and sustainable school furniture aimed at children in the first grade of education from educational institutions with scarce resources in the Bucaramanga Metropolitan Area. In addition, the product was developed as part of a process of appropriation of the object by the user, it was determined that the furniture functions as a playful and mediating object of significant learning in terms of spatial arrangement, ecology, functionality and graphic artistic intervention. In the development of this project, various disciplines were interspersed with the contribution of their own methods such as the philosophy of Lean Startup and ethnography; the design process was carried out according to the design thinking methodology, both in the graphics aspect and in the industrial design. This work was based on the inclusive design for the integral education of children that require attention in their education; the product allows not only to have furniture, but to develop basic cognitive processes for the age of six, the development of skills and logical processes of recognition.

Keywords: graphic design, industrial design, school, furniture, sustainable.

\section{Introducción}

Este estudio partió del presupuesto de desarrollar soluciones al problema de ofrecer opciones de mobiliario a una población específica de niños en edad escolar básica. En el mercado actual colombiano son limitadas las alternativas de mobiliario dirigido a este sector, con el agravante derivado de los altos costos de estos productos, situación que limita la compra a un sector importante de la población.

Con estas premisas, se planteó un proyecto de investigación que propiciara la participación de estudiantes en la valoración y evaluación de los problemas del entorno, y que permitiera el trabajo interdisciplinar de los participantes de los semilleros de investigación Dicubo, que estudia problemas en torno al diseño industrial y el semillero Innume, que se encarga de desarrollos relativos al diseño gráfico, ambos pertenecientes a la Universidad de Investigación y Desarrollo (UDI).

A partir de la pregunta de investigación: ¿Qué características formales y estructurales debe tener un mueble de estudio para niños que cursan primer grado de escolaridad en el Área Metropolitana de Bucaramanga?, se abordó el diseño y construcción de mobiliario que solucionara algunos de los problemas detectados en la exploración preliminar del estudio.

Bajo este marco de ideas, el proyecto se propuso el diseño de un mobiliario escolar portátil y sustentable dirigido a niños en primer grado de escolaridad de instituciones educativas con escasos recursos del Área Metropolitana de Bucaramanga. Además, el producto se planteó como parte de un proceso de apropiación del objeto por parte del usuario; se determinó que el mueble funcionara como objeto lúdico y mediador de aprendizajes significativos en cuanto a ordenación espacial, ecología, funcionalidad e intervención gráfica artística.

\section{Materiales y métodos}

En el desarrollo de este proyecto se entrecruzaron diversas disciplinas con el aporte de sus métodos propios; el proceso de diseño se llevó a cabo de acuerdo a la metodología de design thinking, tanto en el aspecto gráfico como en el diseño industrial, con aportes de la metodología proyectual de Munari, B. (2004), la ingeniería Kansei, la filosofía del Lean Startup y la etnografía (ver Figura 1).

El estudio incorporó la etnografía como herramienta para la caracterización e interpretación de pautas de socialización, la construcción de valores, el desarrollo y las expresiones de la competencia cultural, y el desarrollo y la comprensión de las reglas de interacción de los niños en el aula.

Estas pesquisas ayudaron a determinar aspectos clave como el trabajo en equipo y la distribución espacial en el lugar de estudio; al mismo tiempo, soportaron la construcción de alternativas de apropiación del mobiliario y aportaron a la comprensión del sentido sustentable del producto por parte de los niños.

\section{Población de estudio y selección de la muestra}

\section{Usuarios directos}

Los usuarios directos de este proyecto son los niños con una edad comprendida entre cinco y siete años que cursan primer curso de básica primaria; según el Ministerio de Educación Nacional (MEN) "La Educación Básica comprende un ciclo de educación 'básica primaria' que se extiende del grado 1 al 5, a niños cuyas edades oscilan entre los 6 y los 10 años" (Organización de los Estados Iberoamericanos [OEI], 2006). Si asumimos que los niños están en máximo un año de extraedad, término que define el desfase de edad de un estudiante respecto al promedio esperado para cursar un determinado grado 
(MEN, 2013), tendríamos niños en edades comprendidas entre cinco y siete años.

La educación infantil se definió por primera vez a través del Decreto 2101 de 1939 como "(...) aquella que recibe el niño entre los cinco y siete años, cuyo objetivo principal es crearle hábitos necesarios para la vida, juntamente con el desarrollo armónico de la personalidad" (Cerda, 1996). Los planes educativos se encaminan a "Desarrollar integral y armónicamente sus aspectos biológico, sensomotor, cognitivo y socioafectivo, y en particular la comunicación, la autonomía y la creatividad, y con ello propiciar un aprestamiento adecuado para su ingreso a la Educación Básica" (MEN, 2014).

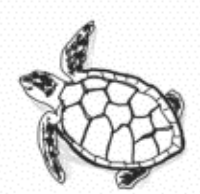

CURVAS

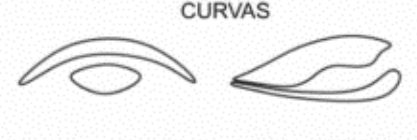

VISTAFRONTAL VISTALATERAL
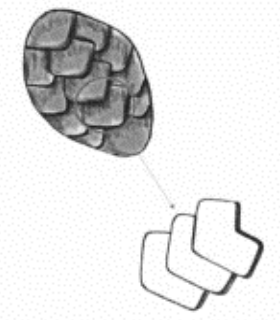
UNIONES ENTRE (TESELACIONES) DE PLACAS
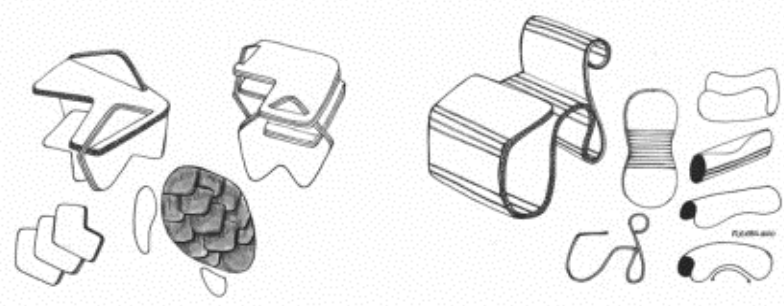

Figura 1. Obtención y desarrollo de los módulos constructivos. Fuente: Autores.

\section{Usuarios indirectos}

Los usuarios indirectos son los padres, los tutores o profesores y las instituciones educativas que pueden tomar la decisión de compra del mobiliario. El proyecto se encaminó a grupos familiares con niños entre los cinco y siete años de estratos uno y dos. La población del departamento de Santander en edad comprendida entre los 5 y los 9 años es de 187525 según la proyección hecha de acuerdo al censo de 2005; además, el 78,0\% de la población de 5 a 6 años asiste a un establecimiento educativo formal (Departamento Administrativo Nacional de Estadísticas, 2006).

Recapitulando, el producto está dirigido a una población meta de 146270 niños que asisten a una institución educativa y se focaliza en los pertenecientes a estratos 1 y 2, es decir, aproximadamente 30000 niños en el Área
Metropolitana de Bucaramanga. Estas condiciones de la población meta explican algunas de las decisiones tomadas en cuanto a materiales y precios posibles del mobiliario en el mercado.

\section{Resultados y discusión}

\section{Producto y concepto}

La sostenibilidad del proyecto considera las dimensiones social, ambiental y económica. Desde lo social, el mobiliario desarrollado presenta características que responden a problemas locales, como el acceso a una infraestructura adecuada para el estudio por parte de los niños de escasos recursos económicos. La economía a escala local fue otro lineamiento que ayudó a determinar el tipo de material y los procesos de manufactura propios de las realidades productivas locales, en consonancia con los postulados de Manzini (2017), sobre la "ecología de lo artificial basada en la valoración sostenible de los recursos locales (entendiéndose tanto recursos físicos como recursos socio - culturales)".

La responsabilidad ambiental guio el proceso de diseño en varios aspectos: primero, al determinar el mobiliario como medio para la ecoalfabetización; en segundo lugar, le dio lineamientos para la materialización de los conceptos a través de herramientas y métodos propios del diseño para el medio ambiente (DFE) como el diseño para el montaje, el desmontaje, y la concepción del objeto a través de sus componentes constitutivos para procurar iguales tiempos de vida en sus componentes, la posibilidad para su mantenimiento y la actualización, entre otras características.

\section{Diseño interdisciplinario}

El proyecto de diseño y construcción del mobiliario escolar, formalizado como diseño con un propósito, diseño para todos o incluyente, se desarrolló como un trabajo interdisciplinar entre los integrantes del semillero Innume de la Escuela de Diseño Gráfico de la UDI, que elaboró el concepto de identidad, estrategia visual del producto y el packaging en asocio con los estudiantes del semillero Dicubo de la Escuela de Diseño Industrial, que trabajaron en los aspectos de ergonomía, antropometría, impacto ambiental y sostenibilidad. El diseño de producto se contempló a la luz de las dos disciplinas, y se abordaron los aspectos de conceptualización, psicología cognitiva y diseño objetual en el marco de los derechos infantiles según la legislación colombiana (Díaz \& Sarmiento, 2014).

\section{Desde la función}


El mobiliario se proyectó pensando en su portabilidad y en optimizar las condiciones de su uso. Para su construcción se utilizó como materia prima el cartón Hexacomb Falconboard, que respondió de manera óptima a las pruebas de resistencia realizadas; además, es un material amigable con el medio ambiente y con certificación Sustainable Forestry Initiative (SFI). En este punto, se resalta que uno de los fundamentos del proyecto fue el concepto de sostenibilidad, que tiene sus inicios en la década de los ochenta. En el Informe Brundtland, la sostenibilidad es definida como "el desarrollo que satisface las necesidades de la generación presente sin comprometer la capacidad de las generaciones futuras para satisfacer sus propias necesidades" (Luffiego \& Rabadán, 2000); i este concepto derivó en el de sostenibilidad fuerte, que se puede definir como la "viabilidad de la relación que mantiene un sistema socioeconómico con un ecosistema" (Naredo, 1994 citado por Luffiego \& Rabadán, 2000). La concepción de sostenibilidad fuerte es ideal pero también utópica, en tanto que aunque procura la conservación de los ecosistemas impediría el crecimiento. Posteriormente, se desarrolló la idea de sostenibilidad integral, en la cual se trata de diferenciar tres tipos de sostenibilidad: ecológica, social y económica. Con estos conceptos, el mobiliario se proyectó con una vida útil de un año para no generar residuos sólidos al ambiente después de cumplir su desgaste máximo.

Se analizaron diferentes tipos de materiales en procura de portabilidad, duración y estética; se buscaba que su estructura otorgue resistencia mecánica, gramaje en el papel y altura de la onda, factores determinantes en la resistencia y la carga mecánica. De esta manera, a partir de las pruebas se determinó que la mejor opción era el cartón definido por Digital Graphic Systems (DGS), como: "Hexacomb Falconboard, sustrato rígido elaborado con en base a material reciclado y $100 \%$ reciclable, consistente en una estructura tipo "panal de abeja" hecha con papel kraft y recubierta con cartulina gruesa en ambas caras. Producido con papel reciclado con mínimo contenido de cloro-uorcarbono, Falconboard emite menos de $1 \%$ de VOC (compuestos volátiles orgánicos). Estas cualidades lo hacen una excelente alternativa "verde" comparado con los sustratos rígidos tradicionales" (DGS, 1993).

El material es un producto reciclable, que se convierte en materia prima secundaria que permite integrarla nuevamente a un ciclo productivo como fibra reciclada.

\section{Módulos y morfogénesis}

De acuerdo con Wong (1993), los módulos son formas idénticas o similares entre sí, que se repiten

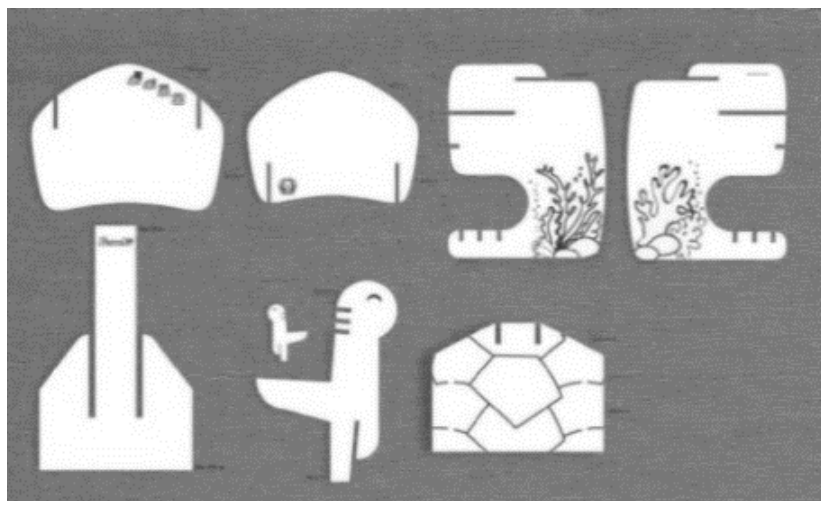

Figura 2. Módulos constructivos. Fuente: Autores.

sistemáticamente en el espacio, y se consideran unidades de medida que determinan las proporciones entre las diferentes partes del diseño (ver Figura 2). La repetición de módulos es uno de los principios constructivos más efectivos en el diseño; en tanto que permite la construcción de formas armónicas y lleva a la unificación de la pieza, "cada módulo que se repite es como el compás de un ritmo dado".

El diseño basado en módulos funcionales posibilita la creación de estructuras mayores que pueden ser dispuestas de diferentes formas; los módulos permiten "optimizar el tiempo de construcción debido a que son transportables, desarmables y reorganizables, permiten impulsar múltiples funcionalidades y su reutilización al generar un nuevo uso diferente al que fueron fabricados" (Meneses, 2017). Además, se utilizó el concepto de morfogénesis u origen controlado de la forma, que, según la propuesta de Sánchez (2009), es planteada "desde una perspectiva histórica, desde una genética del objeto (morfogenética) y, desde otro, por la incidencia de la diseñística como concepción de su origen (morfoconcepción).

En el desarrollo de diseño se revisaron tipologías de identidad visual para la formulación y creación del concepto gráfico como representación del imagotipo de la marca. Finalmente, se definió Ecomobi como nombre del producto; su construcción se hizo siguiendo la metodología de naming planteada por Xavier Grau (Grau, X., 2011). La imagen representa características ecológicas, amigables, divertidas y llamativas e integra en su identidad visual desarrollos gráficos a partir de representaciones de animales colombianos en vía de extinción, como el armadillo gigante, la guacamaya bandera, el manatí del Caribe, el oso perezoso, el mico de noche andino, el caimán del Orinoco y la tortuga carey (ver Figura 3). 


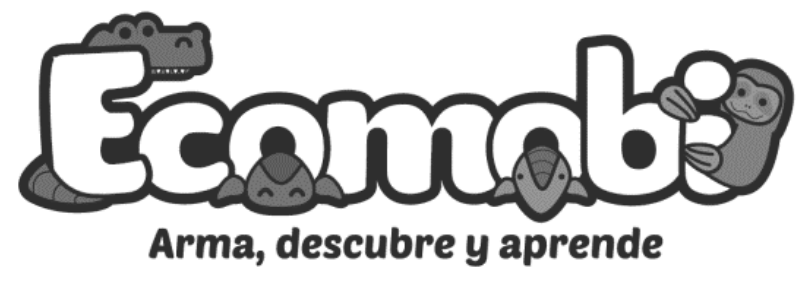

Figura 3. Imagotipo del producto Ecomobi. Fuente: Autores.

\section{Desde la estética}

El concepto gráfico que desarrolló el proyecto giró en torno a la concienciación de los niños acerca de la protección de los animales en vía de extinción en el hábitat colombiano; el diseño del mobiliario planteó conservar la forma de la mesa como un mismo elemento característico del hábitat y protección del animal a reconfigurar en la forma, es decir, la estructura y morfología de la silla bajo mesa funciona como símbolo de protección al simular la guarida del animal, en este caso de estudio, la tortuga de carey.

En el proceso de diseño se construyó un prototipo a escala real para la etapa de comprobación, y se analizaron aspectos como la interacción y el comportamiento de los niños participantes frente a un nuevo producto para ellos. En este punto se contó con el enfoque de una profesional en terapia ocupacional con énfasis en trabajo con niños; se evaluaron aspectos como la comprensión del ejercicio de armar, descubrir y aprender con el análisis directo dado por la observación frente a la experiencia de la comprobación (Simanca, Porras, Garrido \& Hernández, 2017).

Posterior al desarrollo de esta fase de análisis de la forma-función y la incorporación de nuevos materiales se realizó la exploración funcional experimental con la comprobación, en varias ocasiones, del producto, mediante el ejercicio de montarlo y desmontarlo para obtener nuevas alternativas a la estructura formal propuesta. Se partió de la creación de modelos simples que permitieran evaluar los aspectos formales señalados anteriormente, comparando los modelos visuales con los modelos funcionales (ver Figura 4).

\section{Valor diferencial del producto}

Además de lo señalado, este trabajo se fundamentó en el diseño incluyente para la formación integral de niños que requieren atención en su educación; el producto permite no solo contar con un mobiliario portátil, sino desarrollar procesos cognitivos básicos para la edad de seis años, así como de habilidades y procesos lógicos de reconocimiento.
El mobiliario se configura como mediador de valores para comprender la situación actual de los animales colombianos en vía de extinción, los peligros que los acechan, su hábitat, y las razones por las cuales se deben preservar y cuidar. Este proceso se consolida cuando el niño se enfrenta al reto de armar, descubrir y aprender a partir del ensamble del mobiliario y de la personalización a su estilo a partir de habilidades artísticas y técnicas plásticas (ver Figura 5).

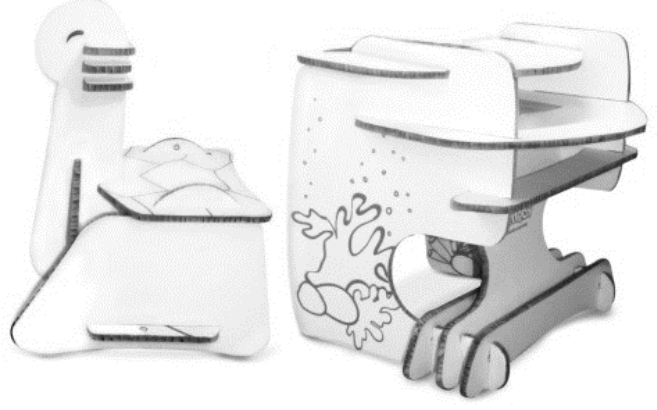

Figura 4. Mobiliario Ecomobi. Fuente: Autores.

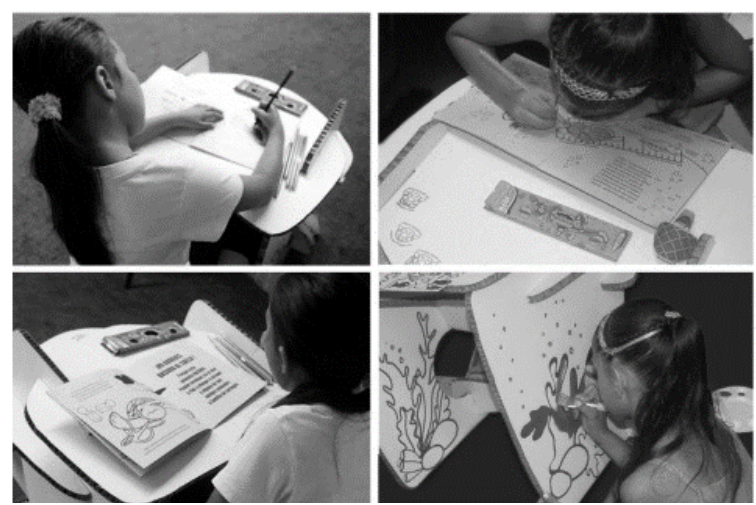

Figura 5. Personalización del mobiliario. Fuente: Autores.

Finalmente, se propuso un packaging en forma de carpeta portable en cuyo interior están las piezas separadas con el indicativo gráfico (ver Figura 6); una vez abierto el producto, se accede al mobiliario organizado por piezas para su armado y ensamble. Los elementos están ordenados de manera ascendente para facilitarle al niño el proceso de armado; los indicadores están dispuestos por números dentro de contenedores con formas particulares que facilitan su ubicación e interpretación.

\section{A modo de conclusión}

El proceso de personalizar y crear su propio espacio de estudio le permite al niño desarrollar competencias, favoreciendo el pensamiento creativo, la imaginación y 
el aprendizaje autónomo; al mismo tiempo, se estimula la colaboración y el desarrollo en equipos de trabajo.

Cabe señalar que esta metodología de trabajo permite al infante mejorar la comunicación a través del proceso constructivo y lo lleva a identificar y resolver problemas que requieren una gestión compleja mediante el conocimiento táctico. Tal como los manifiesta Seumenicht (2014), la metodología del Lego Serious Play evidenció, de acuerdo con estudios científicos, que las células cerebrales están conectadas con las manos. Es decir, nuestras manos son capaces de retener información que muchas veces no podemos expresar de manera verbal. Al construir con las manos conceptos abstractos podemos acceder directo al conocimiento y a la experiencia almacenada en la mente, facilitando el razonamiento y la conceptualización de situaciones, proyectos, ideas o planes.

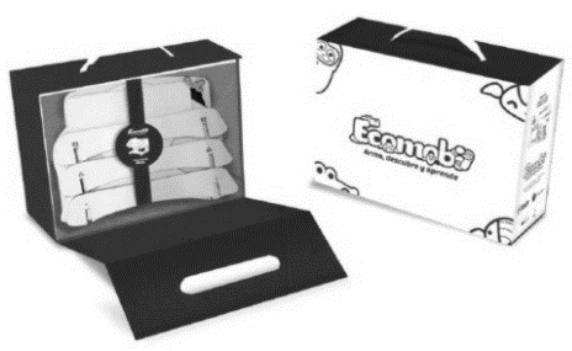

Figura 6. Packaging del mobiliario. Fuente: Autores.

Finalmente, se diseñó una cartilla que acompaña la experiencia con el mobiliario (ver Figura 7), en la cual, a través de actividades propias de la edad escolar del niño, se narra la historia de las tortugas de carey, se describe su hábitat y se les informa del peligro de extinción que afronta esta especie.

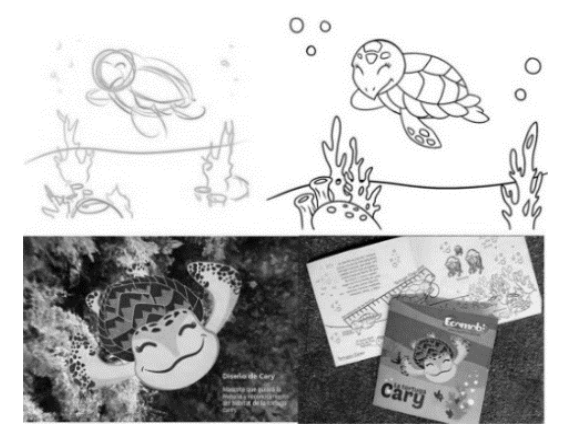

Figura 7. Proceso creativo del diseño de la cartilla. Fuente: Autores.

En futuros desarrollos se plantea la construcción de mobiliario bajo el mismo esquema de trabajo a partir de otros animales en vía de extinción como el armadillo gigante, la guacamaya bandera, el manatí del Caribe, el oso perezoso, el mico de noche andino y el caimán del Orinoco.

\section{Referencias}

Cerda, H. (1996). Educación preescolar: historia, legislación, currículo y realidad socioeconómica. Cooperativa Editorial Magisterio.

Departamento Administrativo Nacional de Estadísticas. (2006). Censo general 2005. Recuperado de https:// www.dane.gov.co/index.php/estadisticas-por-tema/ demografia-y-poblacion/censo-general-2005-1

Díaz, C. H. \& Sarmiento, S. C. (2014). Fundamentos para la gestión del conocimiento en grupos de investigación. I+D Revista de Investigaciones, 3(1), 40-50.

Digital Graphic Systems. (1993). Cartón estructural para impresión digital. Recuperado el 15 de abril de 2015, de https://dgs-usa.com/falconboard-2/

Grau, X. (2011). Naming ¿Cómo crear un buen nombre? Barcelona: UOC.

Luffiego, M. \& Rabadán, J. (2000). La evolución del concepto de sostenibilidad y su introducción en la enseñanza. Historia y epistemología de las ciencias, 18 (3), 473-486.

Manzini, E. (2017). Entrevista a Ezio Manzini. Recuperado el 25 de junio de 2018 de http://www.30-60. com.ar/sec_apuntes_web_detalle.asp?id_ contenido=3ASs8aqkBbtHWKJs\&titulo=apuntes $\% 20$ web $\% 20 . \% 20$ Entrevista $\% 20$ a $\% 20$ Ezio $\% 20$ Manzini\&identificador=apuntesweb

Meneses, M. (2017). Espacios adaptables a través del diseño modular. Pereira: Universidad Católica.

Ministerio de Educación Nacional - MEN. (2014). Sentido de la educación inicial, Documento No. 20. Recuperado el 13 de febrero de 2015, de http://www.colombiaaprende. edu.co/html/familia/1597/articles-341487_doc20.pdf

Ministerio de Educación Nacional - MEN. (2013). Extraedad. Recuperado el 11 de febrero de 2015, de http://www. mineducacion.gov.co/1621/article-82787.html

Munari, B. (2004). ¿Cómo nacen los objetos?: apuntes para una metodología proyectual. Barcelona: Gustavo Gili.

Organización de los Estados Iberoamericanos. (2006). Sistemas Educativos Nacionales-Colombia. Recuperado el 10 de febrero de 2015, de http://www.oei.es/quipu/ colombia/col04.pdf

Sánchez, M. (2009). Morfogenésis del objeto de uso. La forma como hecho social de convivencia. Bogotá: Diseño LA.

Seumenicht, B. (2014). ¿Qué harían las nubes sin imaginación? Revista visión, 14, 31. Recuperado el 24 de febrero de 2015, de http://www.udlap.mx/exaudlap/ files/Revista.pdf

Simanca, F. A., Porras, A. A., Garrido, F. B., \& Hernández, P. C. (2017). Implementación de herramientas tecnológicas en los procesos de enseñanza- aprendizaje de los triángulos. I+D Revista de Investigaciones, 10(2), 79-88. https://doi.org/10.33304/revinv.v10n2-2017006

Wong, W. (1993). Fundamentos del diseño. Barcelona: G. Gili. 